\title{
A Novel Technique for Imaging and Analysis of Hair Cells in the Organ of Corti Using Modified Sca/eS and Machine Learning \\ Shinji Urata ${ }^{1}, 2$, Tadatsune lida ${ }^{1}$, Yuri Suzuki ${ }^{1}$, Shiou-Yuh Lin², Yu Mizushima², Chisato Fujimoto², Yu Matsumoto², Tatsuya Yamasoba2, *
}

\author{
1Department of Cellular Neurobiology, University of Tokyo, Tokyo, Japan; ${ }^{2}$ Department of \\ Otolaryngology, Graduate School of Medicine, University of Tokyo, Tokyo, Japan \\ *For correspondence: tyamasoba-tky@umin.ac.jp; okabe@m.u-tokyo.ac.jp
}

\begin{abstract}
[Abstract] Here, we describe a sorbitol-based optical clearing method, called modified Sca/eS that can be used to image all hair cells (HCs) in the mouse cochlea. This modification of Sca/eS is defined by three steps: decalcification, de-lipidation, and refractive index matching, which can all be completed within $72 \mathrm{~h}$. Furthermore, we established automated analysis programs that perform machine learningbased pattern recognition. These programs generate 1) a linearized image of $\mathrm{HCs}, 2$ ) the coordinates of HCs, 3) a holocochleogram, and 4) clusters of HC loss. In summary, a novel approach that integrates modified Sca/eS and programs based on machine learning facilitates quantitative and comprehensive analysis of the physiological and pathological properties of all HCs.
\end{abstract}

Keywords: Cochlea, Optical tissue clearing, Auto hair cell analysis, ScaleS, Machine learning

[Background] Sound waves reach the inner ear via conduction mechanisms in the external and middle ear. The cochlea is an organ in the inner ear that transduces mechanical stimulation into electronic signals. As a result, generated auditory information is transferred to the brain. Hair cells (HCs) in the organ of Corti, an auditory sensory epithelium, are protected by the temporal bone and are precisely disposed to detect every frequency band from the base (high pitch) to the apex (low pitch) (von Békésy, 1990). Functional analysis of the cochlea is limited due to its anatomical specificity. Surface preparation and sectioning (paraffin or frozen sections) are the most popular methods in histological analysis; however, these techniques do not preserve the three-dimensional structure (Fujimoto et al., 2017; Mizushima et al., 2017). To better understand the processing of auditory information, the location and diversity of HCs must be accurately determined. Consequently, a novel technique to identify intact HCs has long been sought. Established optical clearing methods such as CLARITY, 3DISCO, Sca/eS, iDISCO, and CUBIC (Dodt et al., 2007; Chung et al., 2013; Renier et al., 2014; Susaki et al., 2014; Hama et al., 2015) enable imaging of intact brains and other organs, but are not optimized for imaging of all HCs (Urata et al., 2019). Based on recently developed optical clearing methods for hard tissues such as vDISCO, Bone CLARITY, PEGASOS, CUBIC-X, PACT, and PARS (Calve et al., 2015; Treweek et al., 2015; Berke et al., 2016; Greenbaum et al., 2017; Cai et al., 2018; Jing et al., 2018; Tainaka et al., 2018), we present a sorbitol-based optical clearing method (modified Sca/eS) that is optimized for intact imaging of all mouse HCs in the cochlea (Urata et al., 2019). 
By acquiring successive images of HCs using modified Sca/eS, we established a novel algorithm for comprehensive analysis of HCs. These programs are available on GitHub (https://github.com/okabelab/cochlea-analyzer.git). Use of modified Sca/eS in combination with these programs facilitates quantitative and comprehensive analysis of the physiological and pathological properties of all HCs.

\section{Materials and Reagents}

1. Blu Tack (Bostik, catalog number: 371351 )

2. Glass microscope slide (Matsunami, catalog number: S024410)

3. Glass microscope coverslip (Matsunami, catalog number: C018181)

4. Basukoku (Cemedine; silicone-based adhesive, catalog number: HJ-148)

5. Alexa Fluor 488-conjugated goat anti-rabbit IgG $(\mathrm{H}+\mathrm{L})$ (Life Technologies, catalog number: 1937195)

6. 4\% paraformaldehyde diluted in phosphate-buffered saline (PBS) (Wako, catalog number: 16320145)

7. Triton $X-100$ (Nacalai-Tesque, catalog number: 12967-45)

8. Urea (SIGMA, catalog number: U0631-1KG)

9. Guanidine hydrochloride (Nacalai-Tesque, catalog number: 17318-82)

10. D-sorbitol (SIGMA, catalog number: S1816-1KG)

11. D-glucose (SIGMA, catalog number: G8270-100G)

12. Rabbit polyclonal anti-myosin VIla antibody (Proteus Biosciences, catalog number: 25-6790)

13. $10 \%$ EDTA-2Na (Muto Pure Chemicals, catalog number: 85653 )

14. $\mathrm{NaCl}$

15. $\mathrm{KCl}$

16. $\mathrm{Na}_{2} \mathrm{HPO}_{4} \cdot 12 \mathrm{H}_{2} \mathrm{O}$

17. $\mathrm{KH}_{2} \mathrm{PO}_{4}$

18. Solution 1 (see Recipes)

19. Solution 2 (see Recipes)

20. PBS (see Recipes)

\section{Equipment}

1. Dumont tweezers, straight (World Precision Instruments, catalog number: 500233)

2. Dumont tweezers, straight (World Precision Instruments, catalog number: 14098)

3. Stereoscopic microscope (for microdissection), e.g., SMZ-2B (Nikon)

4. Fluorescence microscope

Confocal microscope (for imaging at a depth of up to 300-500 $\mu \mathrm{m}$ ), e.g., A1MP confocal microscope (Nikon)

Multiphoton microscope (recommended for deeper imaging), e.g., A1MP two-photon 
microscope (Nikon)

5. Objective lenses (long working distance objectives), e.g., N25X-APO-MP (NA1.10, WD2.00) for multiphoton imaging and VC 20x (NA0.75, WD1.00) for confocal imaging

6. Water bath at $37^{\circ} \mathrm{C}$

7. Fridge at $4{ }^{\circ} \mathrm{C}$

\section{$\underline{\text { Software }}$}

1. Microsoft Excel (Microsoft)

2. MATLAB (MathWorks, version R2017b)

Image Processing Toolbox

Statistics and Machine Learning Toolbox

Neural Network Toolbox

\section{Procedure}

A. Modified Sca/eS protocol (see Figure 1)
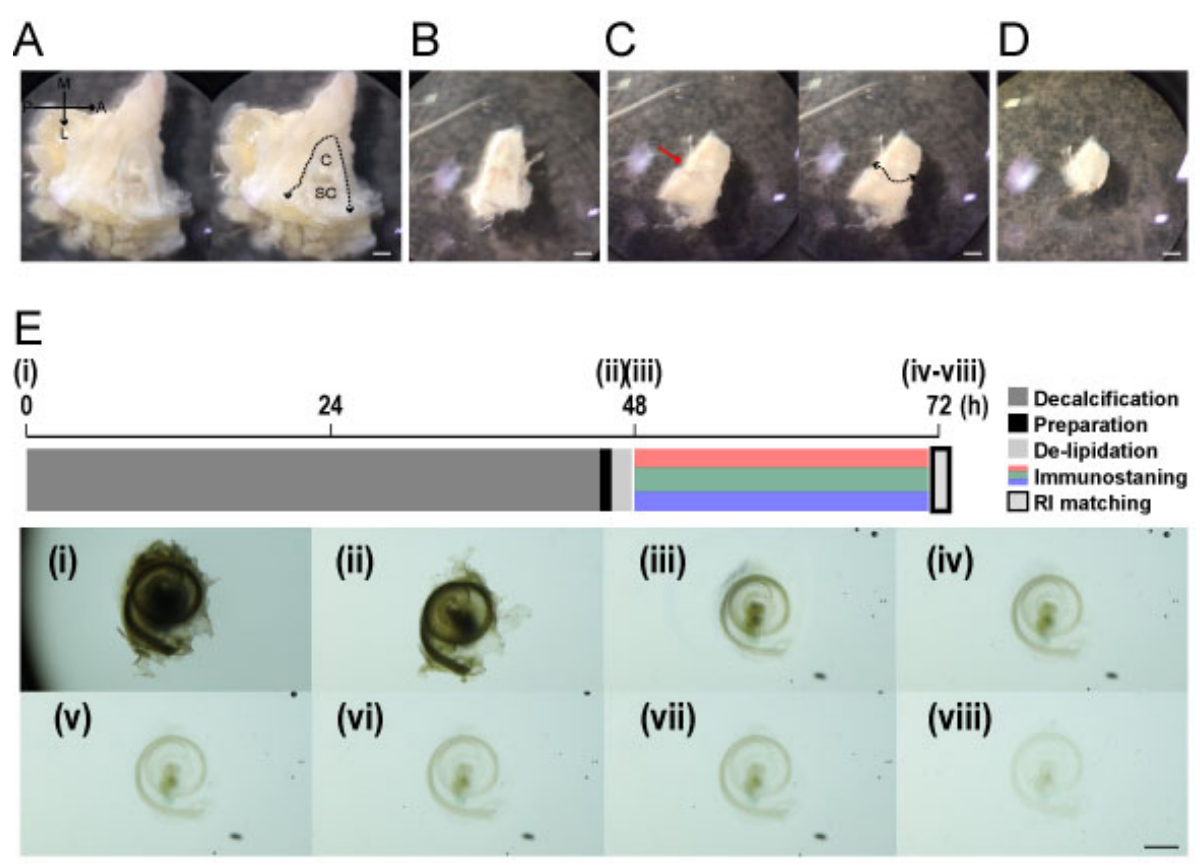

Figure 1. Time course of modified Sca/eS. The inner ear embedded in the temporal bone was removed (panel $A$ ) and dissected along the fistula (B) (dotted lines in the left of panel $A$ ). The cochlea was separated (dotted line in panel $C$ ) from the semicircular canals along the fissure (red arrow in panel C). The cochlea was removed [panel D and (i)] and decalcified (ii). The sample was cleared immediately after being submerged in solution 1 (iii). The sample was then submerged in solution 2 and its transparency gradually increased (iv-viii). A: anterior, P: 
posterior, M: medial, L: lateral, C: cochlea, SC: semicircular canal, RI: refractive index. Scale bar: $1 \mathrm{~mm}$.

1. Extraction of the cochlea from the temporal bone

a. Fix the cochlea in $4 \%$ paraformaldehyde prepared in PBS overnight at $4{ }^{\circ} \mathrm{C}$ with gentle shaking.

b. Wash the cochlea three times (15 min each) with PBS.

c. Incubate the samples in $500 \mathrm{mM}$ EDTA prepared in PBS for $48-120 \mathrm{~h}$ at $37^{\circ} \mathrm{C}$.

Note: The incubation duration depends on the sample size. For example, $48 \mathrm{~h}$ for pups, 72 $h$ for young (2-week-old) mice, and 120 h for middle-age (6-month-old) mice.

d. Wash the cochlea three times (15 min each) with PBS.

e. Remove excess bone tissue surrounding the cochlea.

f. Remove the vestibule and semicircular canal.

2. Tissue de-lipidation (see Figure 1 and Solution 1 in Recipes) Incubate the samples in Solution 1 for $2 \mathrm{~h}$ at $37^{\circ} \mathrm{C}$.

3. Antibody staining (see Figure 1)

a Wash the samples with PBS containing $0.1 \%$ Triton $\mathrm{X}-100$ for 30 min with continuous rocking at $40 \mathrm{rpm}$.

b Incubate the samples in a solution containing the appropriate dilutions of primary antibodies for $2-48 \mathrm{~h}$ at $37^{\circ} \mathrm{C}$.

c Wash the samples three times (30 min each) with PBS containing $0.1 \%$ Triton X-100 with continuous rocking at $40 \mathrm{rpm}$.

d Detect primary antibodies by incubating the samples with a solution containing secondary antibodies for $12-48 \mathrm{~h}$ at $37{ }^{\circ} \mathrm{C}$ followed by three washes with PBS containing $0.1 \%$ Triton $\mathrm{X}-100$.

4. Sample preparation for imaging (see Figures 2 and 3 )

a. Roll up a piece of Blu Tack into a cylindrical shape that is slightly thicker than the cochlea [Figure 2 (i)].

b. Align Basukoku in a horseshoe shape on the glass slide [Figure 2 (ii)].

c. Place the Blu Tack on the Basukoku [Figure 2 (iii, iv)].

d. Place a drop of Basukoku inside the Blu Tack horseshoe to make a pedestal for the cochlea [Figure $2(\mathrm{v})$ ].

e. Carefully place the cochlea on the pedestal [Figure 2 (vi)].

Note: Position the cochlea so that the axis of the center of the cochlea is aligned perpendicular to the glass slide (Figure 2).

f. Place the cover glass on the surface of the Blu Tack and gently push it toward the surface of the cochlea [Figure 2 (vii)].

Note: Keep pushing the cover glass until it reaches the surface of the cochlea. This process is critical to image the whole cochlea [Figure 2 (vii-a)]. 
g. With the horseshoe opening facing upward, add $100 \mu \mathrm{l}$ of solution 2 (see Recipes) to the gap within the horseshoe until the imaging chamber is filled [Figure 2 (viii)].

Note: Images iv-viii in Figure 1 show how transparent the cochlea becomes after 20 min (images were acquired at increments of $5 \mathrm{~min}$ ).

h. Fill the gap between the Blu Tack with Basukoku to seal the horseshoe opening [Figure 2 (ix)].

Note: Air bubbles should not remain in the chamber; this would indicate potential leaking of solution 2 and cause the sample to dry out.
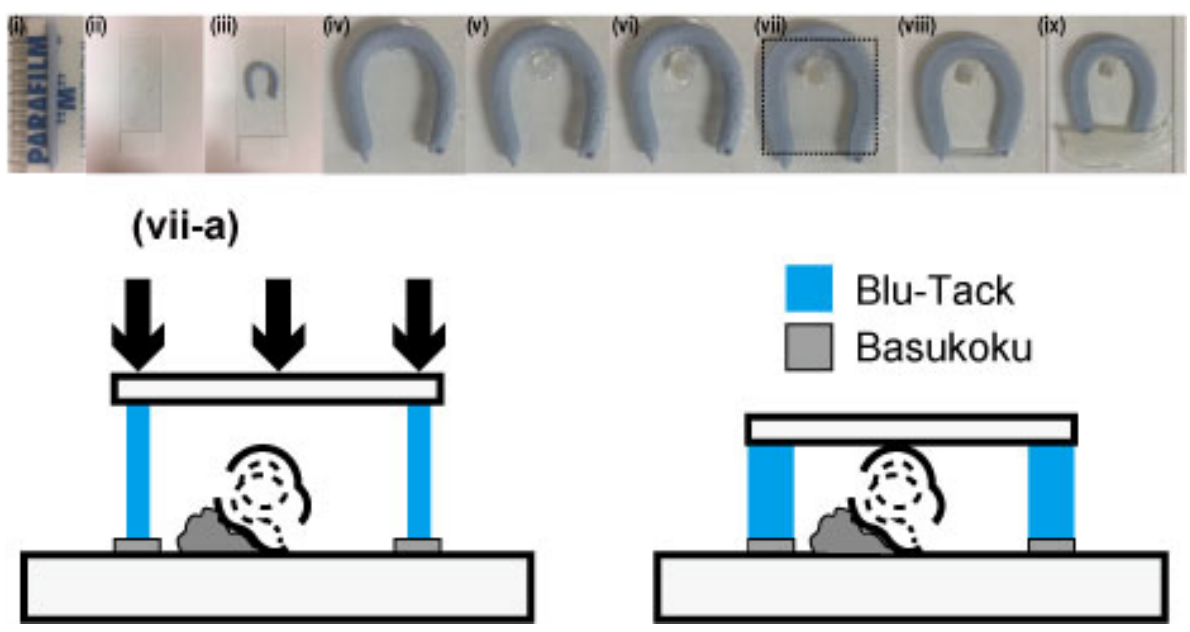

Figure 2. Sample preparation for the imaging step

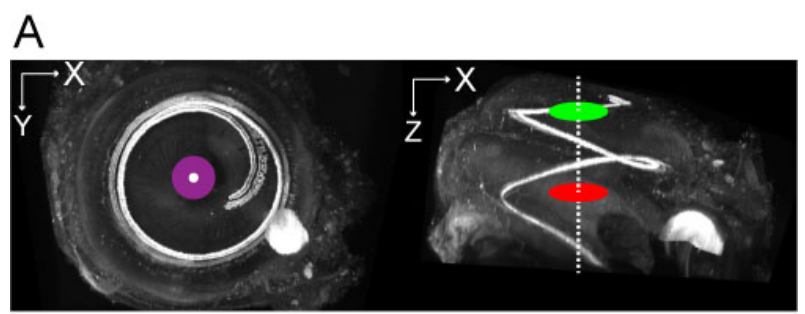

$\mathrm{B}$

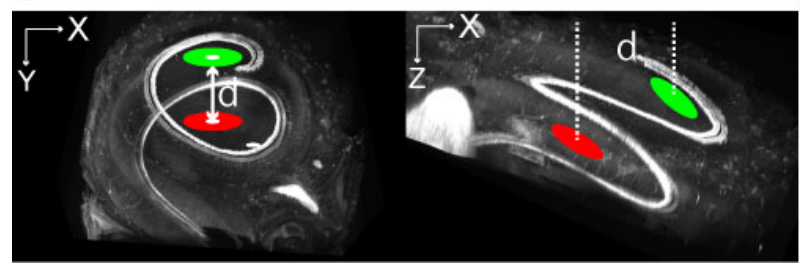

Figure 3. Cochlea position. The center of the first (green) and second (red) turns of the cochlea. Images on the left are the ideal position for imaging of HCs in the whole cochlea, where $d$ represents the distance between the centers of the green and red areas. Upper panels $(A)$ show all HCs imaged using a two-photon microscope in a successful case. Lower panels (B) are representative cases where the approach failed. Images on the left are from the top view, while images on the right are from the side view. The modiolus of the cochlea should be perpendicular to the slide glass [i.e., centers should overlap (purple)]. 
5. Imaging of the sample

a. Place one drop of distilled water on the cover glass and then place the objective lens against the cover glass.

Note: Use an appropriate immersion media for high-resolution imaging. Water-immersion lenses perform much better than air-immersion objective lenses.

b. To image the whole cochlea, images should be successively acquired with $10-40 \%$ overlap [Figure 2 (i)].

c. See example images of sample processed with previous well known clearing methods (Figure 4).

A

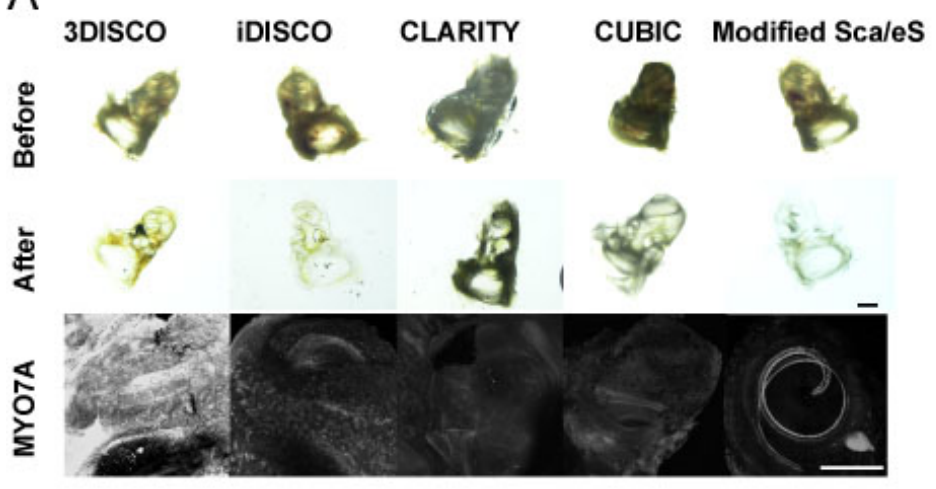

B

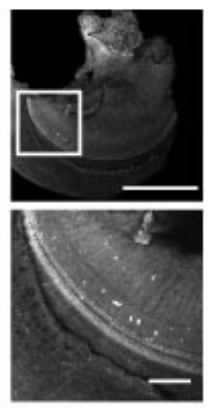

Figure 4. Comparison with other clearing methods. MYO7A-immunopositive $\mathrm{HCs}$ in samples were not detected by 3DISCO, iDISCO, CLARITY, or CUBIC (A). Microdissection of the membrane labyrinth of the iDISCO-processed samples in panel A confirmed the presence of MYO7A-immunopositive $\mathrm{HCs}$, suggesting that the surrounding bone tissue prevented the detection of fluorescence (B). Scale bars: $500 \mu \mathrm{m}$ in panel A and upper image of panel B, 100 $\mu \mathrm{m}$ in lower image of panel $\mathrm{B}$.

B. Automated $\mathrm{HC}$ analysis protocol based on machine learning

HCs were automatically detected and analyzed using custom MATLAB scripts (R2017b, MathWorks), the details of which were provided in a previous study (Urata et al., 2019). The MATLAB source code is available on GitHub (https://github.com/okabe-lab/cochlea-analyzer.git). Data analysis procedures including statistical analysis were described in the original publication of the protocol (Urata et al., 2019). The actual running of the program was shown in this protocol paper.

1. The main $0 . \mathrm{m}$ program ( $1 \mathrm{~min}$ ) checks the integrity of the software so that automated analysis can be flawlessly performed (see Video 1).

Note: If an error occurs during the process, double check to see if any required files are absent under "Function Files" on GitHub (https://github.com/okabe-lab/cochlea-analyzer.git). 


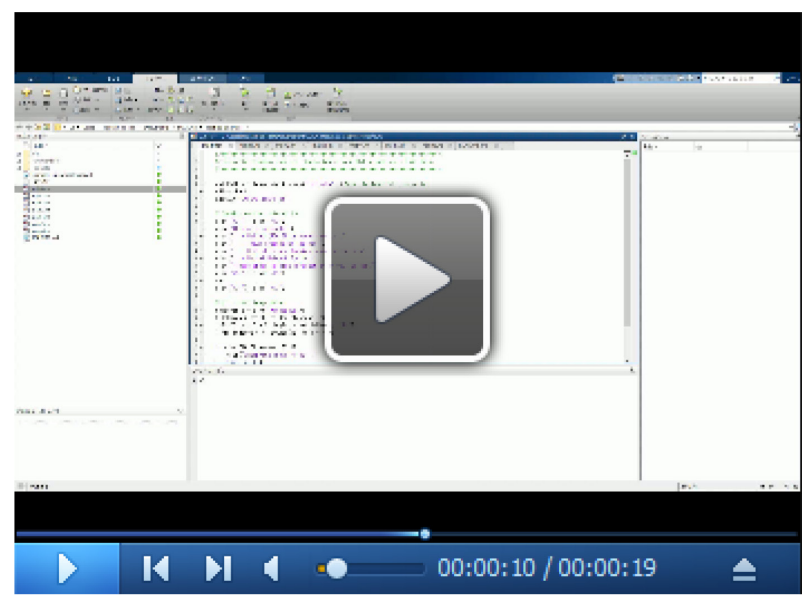

Video 1. Checking the integrity of the software before performing automated analysis using main $0 . m$

2. The main $1 . m$ program merges all the files together to create a linearized image of $\mathrm{HCs}$, which takes about 15 min in total (Step B2 in Figure 5 and see Video 2).

Note: If the main0.m program completes without an error, it is guaranteed to function properly. A TIFF image is produced in this step.

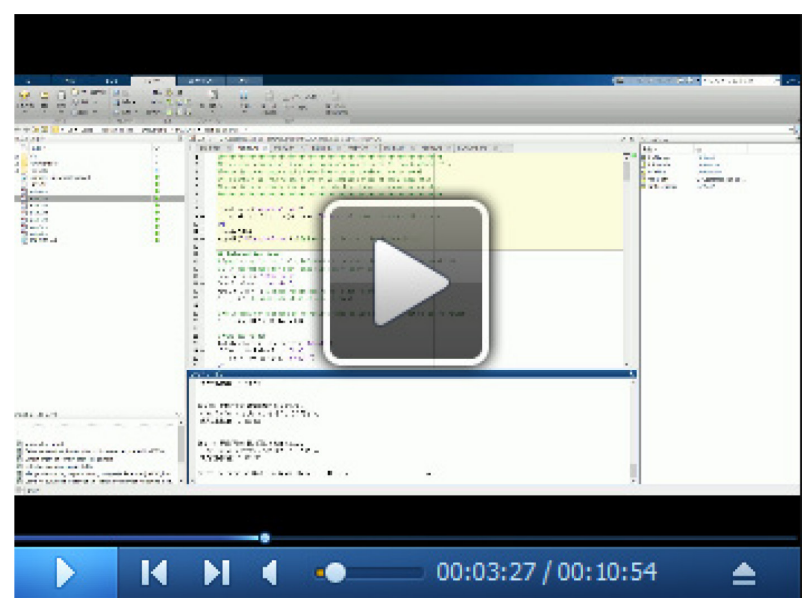

Video 2. Merging of all the acquired files together to create a linearized image of HCs using main $1 . \mathrm{m}$ 
Step 1

Raw data
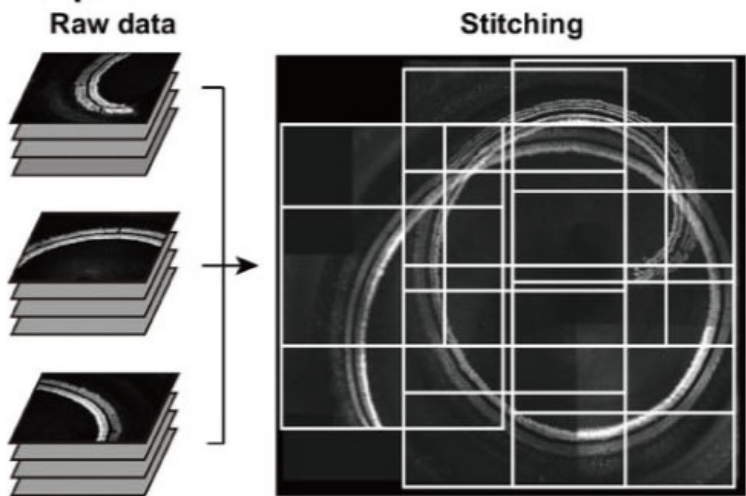

Step 2
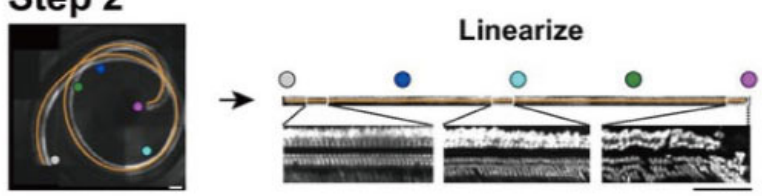

Step 3

Step 4

IHC counting

\section{OHC \& cell loss counting}
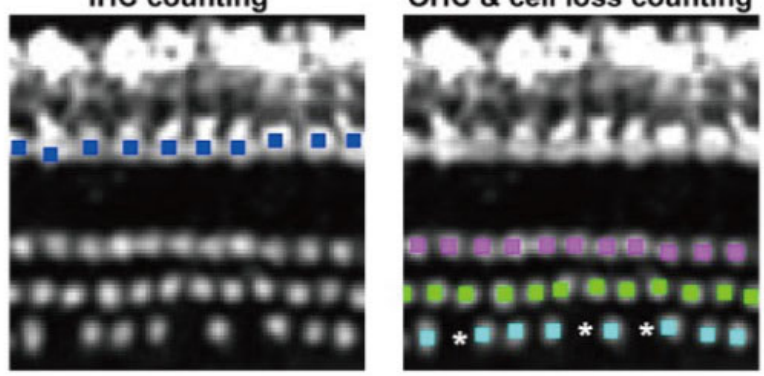

Figure 5. Image generation by the programs. Raw images are stitched (Step 1), and the lines between inner and outer HCs are illustrated (orange line in Step 2). Finally, HCs are linearized and representative images of the apical, middle, and basal portions are enlarged. Colored dots in Step 3 and Step 4 indicate the presence of HCs, and asterisks represent loss of HCs.

3. The main2.m (inner HCs, duration 5 min, see Video 3) and main3.m (outer HCs, duration 3 min, see Video 4) programs detect $169 \mathrm{HCs}$.

Notes:

a. An EXCEL file containing the coordinates of recorded (or imaged) HCs is generated in this step.

b. Advanced analysis is available using the main4-6.m programs. The main4.m program analyzes ( $<1 \mathrm{~min}$ ) the spatial distribution of outer HCs and the main5. m program generates (<1 min) a holocochleogram (see Video 5). The main6.m program analyzes (10 min) clusters of HC loss (see Video 6). 


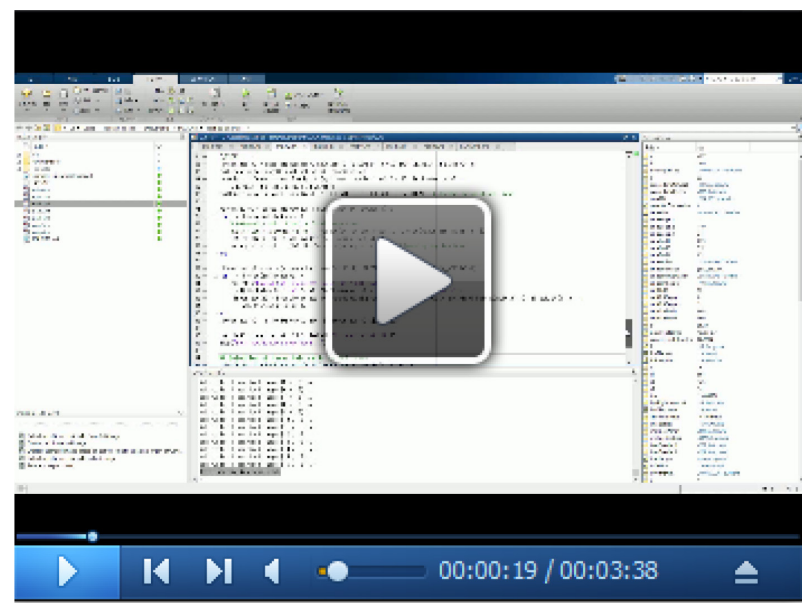

Video 3. Imaging of inner HCs using the main2.m program

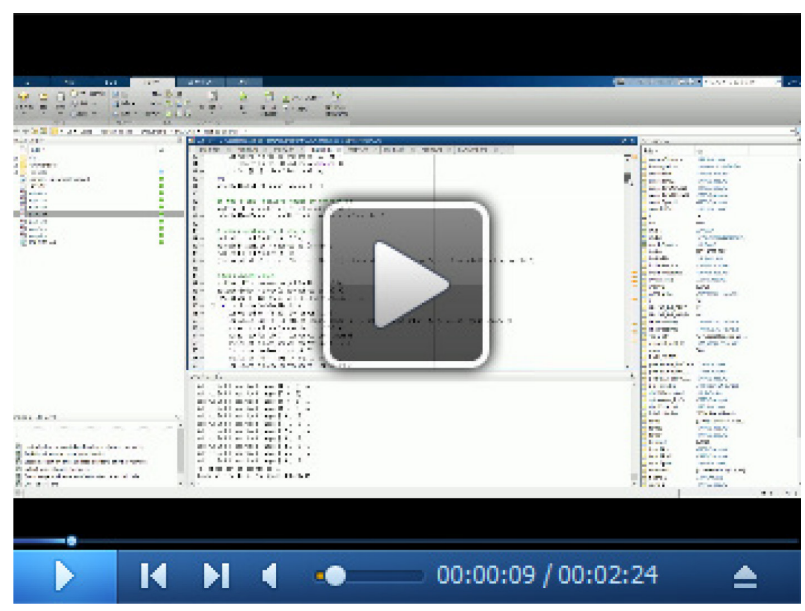

Video 4. Imaging of outer HCs using the main3.m program

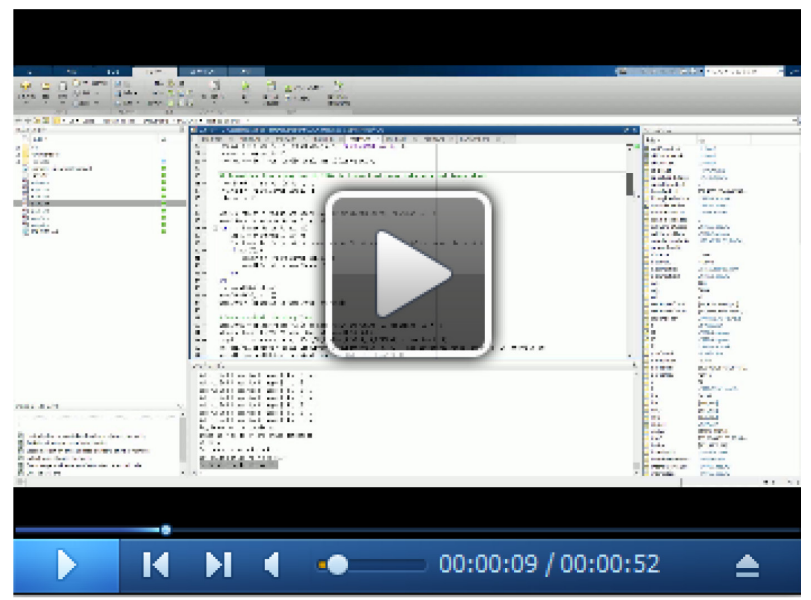

Video 5. Analysis of the spatial distribution of outer HCs using the main4.m program and generation of a holocochleogram using the main $5 . \mathrm{m}$ program 


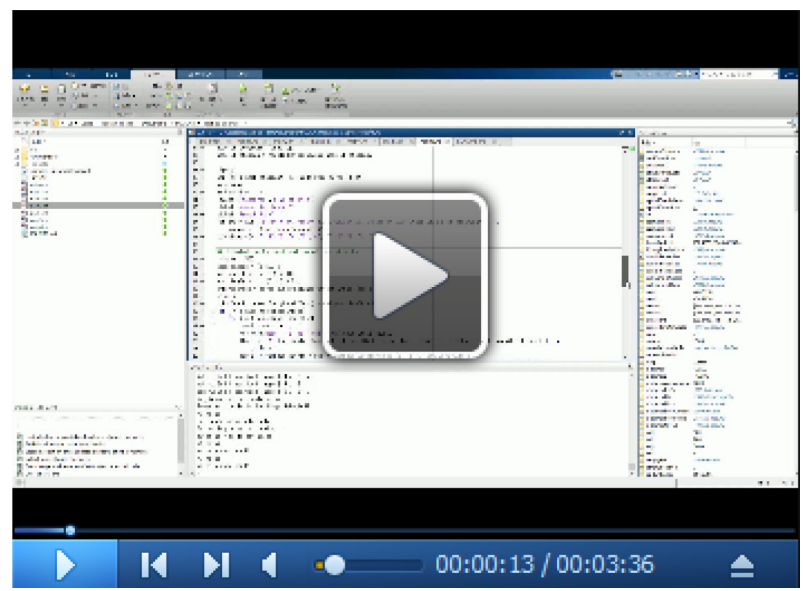

Video 6. Analysis of clustered HC loss using the main6.m program

\section{$\underline{\text { Recipes }}$}

1. Solution $1(20 \mathrm{ml})$

$5.7 \mathrm{~g}$ of guanidinium chloride

$7 \mathrm{~g}$ of D-sorbitol

$3 \mathrm{~g}$ of D-glucose

$800 \mu \mathrm{l}(\mathrm{w} / \mathrm{v})$ of Triton X-100 in PBS (pH 6.0-8.0)

2. Solution $2(20 \mathrm{ml})$

$5.7 \mathrm{~g}$ of guanidinium chloride (or $4.8 \mathrm{~g}$ of urea)

$12 \mathrm{~g}$ of D-sorbitol

$20 \mu$ of Triton X-100 in PBS (pH 7.1)

Note: The solution can be kept at room temperature for up to 2 months for good clearing.

3. PBS

$80 \mathrm{~g}$ of $\mathrm{NaCl}$

$2 \mathrm{~g}$ of $\mathrm{KCl}$

$29 \mathrm{~g}$ of $\mathrm{Na}_{2} \mathrm{HPO}_{4} \cdot 12 \mathrm{H}_{2} \mathrm{O}$

$2 \mathrm{~g}$ of $\mathrm{KH}_{2} \mathrm{PO}_{4}$

Add distilled $\mathrm{H}_{2} \mathrm{O}$ to prepare $1 \mathrm{~L}$ of $10 x$ PBS

Dilute 10-fold with distilled $\mathrm{H}_{2} \mathrm{O}$ to prepare 1x PBS

\section{Acknowledgments}

We thank Dr. Shigeo Okabe for helpful suggestions and supervision. This method was adapted from our original publication of the protocol (Urata et al., 2019). This work was supported by grants from the Japan Society for the Promotion of Science KAKENHI (15K10743, 2653081, and 16K15717), the Japan Science and Technology Agency (JPMJCR14W2), the Japan Agency for Medical Research and Development (17gm5010003), the Japan Society for the Promotion of Science 
Please cite this article as: Urata et. al., (2019). A Novel Technique for Imaging and Analysis of Hair Cells in the Organ of Corti Using Modified Sca/eS and

(17H01387), the UTokyo Center for Integrative Science of Human Behavior, and the Ministry of Education, Culture, Sports, Science, and Technology (18H04727 and 26111506).

\section{Competing interests}

The authors have no conflicts of interest to declare.

\section{$\underline{\text { References }}$}

1. Berke, I. M., Miola, J. P., David, M. A., Smith, M. K. and Price, C. (2016). Seeing through musculoskeletal tissues: improving in situ imaging of bone and the lacunar canalicular system through optical clearing. PloS One 11(3): e0150268.

2. Cai, R., Pan, C., Ghasemigharagoz. A., Todorov, M. I., Foerstera, B., Zhao, S., Bhatia, H. S., Mrowka, L., Theodorou, D., Rempfler, M., Xavier, A., Kress, B. T., Benakis, C., Liesz, A., Menze, B., Kerschensteiner, M., Nedergaard, M. and Erturk, A. (2018). Panoptic vDISCO imaging reveals neuronal connectivity, remote trauma effects and meningeal vessels in intact transparent mice. BioRxiv. doi:10.1101/374785.

3. Calve, S., Ready, A., Huppenbauer, C., Main, R. and Neu, C. P. (2015). Optical clearing in dense connective tissues to visualize cellular connectivity in situ. PLoS One 10(1): e0116662.

4. Chung, K., Wallace, J., Kim, S. Y., Kalyanasundaram, S., Andalman, A. S., Davidson, T. J., Mirzabekov, J. J., Zalocusky, K. A., Mattis, J., Denisin, A. K., Pak, S., Bernstein, H., Ramakrishnan, C., Grosenick, L., Gradinaru, V. and Deisseroth, K. (2013). Structural and molecular interrogation of intact biological systems. Nature 497(7449): 332-337.

5. Dodt, H. U., Leischner, U., Schierloh, A., Jahrling, N., Mauch, C. P., Deininger, K., Deussing, J. M., Eder, M., Zieglgansberger, W. and Becker, K. (2007). Ultramicroscopy: three-dimensional visualization of neuronal networks in the whole mouse brain. Nat Methods 4(4): 331-336.

6. Fujimoto, C., Iwasaki, S., Urata, S., Morishita, H., Sakamaki, Y., Fujioka, M., Kondo, K., Mizushima, N. and Yamasoba, T. (2017). Autophagy is essential for hearing in mice. Cell Death Dis 8(5): e2780.

7. Greenbaum, A., Chan, K. Y., Dobreva, T., Brown, D., Balani, D. H., Boyce, R., Kronenberg, H. M., McBride, H. J. and Gradinaru, V. (2017). Bone CLARITY: Clearing, imaging, and computational analysis of osteoprogenitors within intact bone marrow. Sci Transl Med 9(387). doi: 10.1126/scitransImed.aah6518.

8. Hama, H., Hioki, H., Namiki, K., Hoshida, T., Kurokawa, H., Ishidate, F., Kaneko, T., Akagi, T., Saito, T., Saido, T. and Miyawaki, A. (2015). ScaleS: an optical clearing palette for biological imaging. Nat Neurosci 18(10): 1518-1529.

9. Jing, D., Zhang, S., Luo, W., Gao, X., Men, Y., Ma, C., Liu, X., Yi, Y., Bugde, A., Zhou, B. O., Zhao, Z., Yuan, Q., Feng, J. Q., Gao, L., Ge, W. P. and Zhao, H. (2018). Tissue clearing of both hard and soft tissue organs with the PEGASOS method. Cell Res 28(8): 803-818. 
10. Mizushima, Y., Fujimoto, C., Kashio, A., Kondo, K. and Yamasoba, T. (2017). Macrophage recruitment, but not interleukin 1 beta activation, enhances noise-induced hearing damage. Biochem Biophys Res Commun 493(2): 894-900.

11. Renier, N., Wu, Z., Simon, D. J., Yang, J., Ariel, P. and Tessier-Lavigne, M. (2014). iDISCO: a simple, rapid method to immunolabel large tissue samples for volume imaging. Cell 159(4): 896910.

12. Susaki, E. A., Tainaka, K., Perrin, D., Kishino, F., Tawara, T., Watanabe, T. M., Yokoyama, C., Onoe, H., Eguchi, M., Yamaguchi, S., Abe, T., Kiyonari, H., Shimizu, Y., Miyawaki, A., Yokota, H. and Ueda, H. R. (2014). Whole-brain imaging with single-cell resolution using chemical cocktails and computational analysis. Cell 157(3): 726-739.

13. Tainaka, K., Murakami, T. C., Susaki, E. A., Shimizu, C., Saito, R., Takahashi, K., HayashiTakagi, A., Sekiya, H., Arima, Y., Nojima, S., Ikemura, M., Ushiku, T., Shimizu, Y., Murakami, M., Tanaka, K. F., lino, M., Kasai, H., Sasaoka, T., Kobayashi, K., Miyazono, K., Morii, E., Isa, T., Fukayama, M., Kakita, A. and Ueda, H. R. (2018). Chemical landscape for tissue clearing based on hydrophilic reagents. Cell Rep 24(8): 2196-2210 e2199.

14. Treweek, J. B., Chan, K. Y., Flytzanis, N. C., Yang, B., Deverman, B. E., Greenbaum, A., Lignell, A., Xiao, C., Cai, L., Ladinsky, M. S., Bjorkman, P. J., Fowlkes, C. C. and Gradinaru, V. (2015). Whole-body tissue stabilization and selective extractions via tissue-hydrogel hybrids for highresolution intact circuit mapping and phenotyping. Nat Protoc 10(11): 1860-1896.

15. Urata, S., lida, T., Yamamoto, M., Mizushima, Y., Fujimoto, C., Matsumoto, Y., Yamasoba, T. and Okabe, S. (2019). Cellular cartography of the organ of Corti based on optical tissue clearing and machine learning. Elife 8: e40946.

16. von Békésy, G. (1990). Experiments in hearing. J Acoust Soc Am 88: 2905. 